\title{
Computational study of variations in gap size for the electrostatic levitating force of MEMS device using dual BEM
}

\author{
Shiang-Woei Chyuan ${ }^{\mathrm{a}, *}$, Yunn-Shiuan Liao ${ }^{\mathrm{a}}$, Jeng-Tzong Chen ${ }^{\mathrm{b}}$ \\ ${ }^{a}$ Department of Mechanical Engineering, National Taiwan University, Taipei, Taiwan, ROC \\ ${ }^{\mathrm{b}}$ Department of Harbor and River Engineering, National Taiwan Ocean University, Keelung, Taiwan, ROC
}

Received 31 March 2004; revised 22 May 2004; accepted 1 June 2004

Available online 23 July 2004

\begin{abstract}
For MEMS combdrive performance, the calculation of levitating force due to electrostatic field is very important, and an accurate electrostatic analysis is essential. Because the gap size between combdrive fingers and ground plane or movable finger and fixed finger, plays a very important role for levitation, a study of the effect of gap size variation is indispensable. For diverse gaps of MEMS comdrive design, the dual BEM (DBEM) has become a better method than the domain-type FEM because DBEM can provide a complete solution in terms of boundary values only, with substantial saving in modeling effort, hence the DBEM was used to simulate the fringing of field around the edges of the fixed finger and movable finger of MEMS combdrive for diverse gap size. Results show that the less the gaps between combdrive fingers and ground plane are, the larger the levitating force acting on the movable finger is. In addition, the levitating force becomes more predominant as the gaps between movable finger and fixed finger decrease. By way of DBEM presented in this article, an accurate electrostatic field can be obtained, and the follow-up control method of levitation force for MEMS combdrive can be implemented more precisely.
\end{abstract}

(C) 2004 Elsevier Ltd. All rights reserved.

Keywords: MEMS; Levitating force; Electrostatic; DBEM; FEM; MEMS; Combdrive

\section{Introduction}

A microelectromechanical systems (MEMS) combdrive as shown in Fig. 1 usually has two sets of fingers. The one which is connected to the substrate is called fixed fingers (stationary electrode), and the other which is released from the substrate is called movable fingers. When two different voltages are applied to these two sets of fingers, the resulting electrostatic force drives the movable fingers towards the fixed ones. Thus, motion is produced by this combdrive in the direction of the movement of the movable fingers [1]. Because combdrive can be designed for either electrostatic actuator or capacitive sensing, it has become a very important device in MEMS [2]. Basically the in-plane interdigitated combdrives are used in in-plane or small outof-plane/torsional motions [3], and the asymmetric combdrives can be utilized to generate large out-of-plane or torsional motions [4]. Generally speaking, in a typical

\footnotetext{
* Corresponding author. Fax: +886-347-13318.

E-mail address: yeaing@iris.seed.net.tw (S.-W. Chyuan).
}

in-plane interdigitated combdrive, the capacitance is linear with displacement, resulting in an electrostatic driving force, which is independent of the position of the movable fingers except at the ends of the range of travel [5]. But for some special applications, combdrive with variable-gap profiles can be designed that will deliver desired driving force profiles by solving an appropriate inverse problem [6].

For in-plane interdigitated combdrives, some advantages [2] could be found: (1) Vibrational amplitudes over $10 \mu \mathrm{m}$ are possible with relatively high quality factors at atmospheric pressure, in contrast to structures which move normal to the surface of the substrate. (2) The combdrive capacitance is linear with displacement, resulting in an electrostatic drive force, which is independent of vibrational amplitude. (3) Flexibility in the design of the suspension for the resonator. Though surface-micromachined polysilicon resonators, which are driven by interdigitated capacitors, have several aforementioned attractive properties, it is essential that both movable finger and fixed finger of combdrive remain coplanar for high quality MEMS devices. It was reported that $2 \mu \mathrm{m}$-thick polysilicon resonators with 


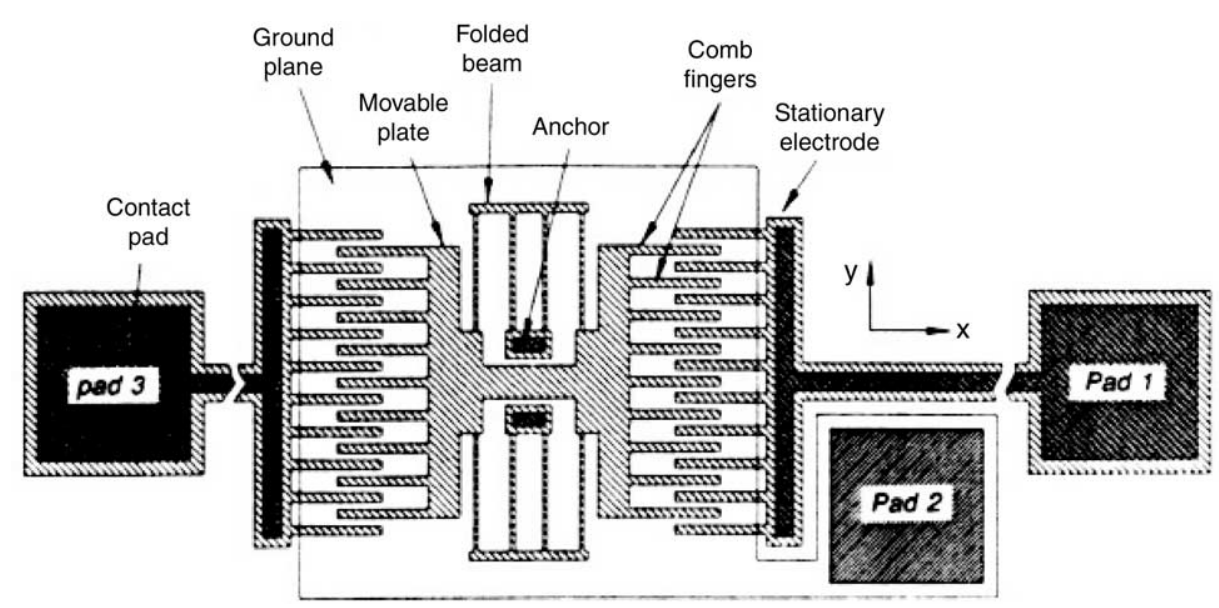

Fig. 1. Layout of a linear lateral resonator driven and sensed with interdigitated capacitors (electrostatic combdrive).

compliant folded-beam suspensions have been observed to levitate over $2 \mu \mathrm{m}$ when driven by an electrostatic comb biased with a DC voltage of $30 \mathrm{~V}$ [2]. Because the levitation effect will seriously downgrade the performance and reliability of MEMS devices, how to obtain the actual electrostatic force responsible for levitation plays a very important role. As a result, knowledge of the electric potential $V$ and electric field intensity $E$ around fixed finger, movable finger and ground plane of MEMS under diverse values of gap for combdrive considering the fringing of field around the edges are very important for engineers because the levitating force acting on the movable finger is obvious dependent on the electrostatic field [7].

Basically, electrical engineers are familiar with electrostatic problems, and diverse numerical methods have been regularly used in MEMS and EM (electromagnetics). Among diverse numerical approaches, finite element method (FEM), which is based on the representation and approximate solution of boundary value problems of engineering mathematics in terms of partial differential equations [8,9], and boundary element method (BEM) based on integral equations [10] have moved from being research tools for scientists to become powerful design tools for engineers. One of the main advantages of BEM, when compared to FEM, is that discretizations are restricted only to the boundaries, making data generation much easier. The BEM is also ideally suited to the analysis of external problems where domains extend to infinity, since discretizations are confined to the internal boundaries with no need to truncate the domain at a finite distance and impose artificial boundary conditions, and to problems involving some form of discontinuity or singularity, due to the use of singular fundamental solutions as test functions. It is also interesting to point out that the unknowns in BEM are a mixture of the potential and its normal derivative, rather than the potential only as in FEM. This is a consequence of the BEM being a 'mixed' formulation, and constitutes an important advantage over FEM. Especially for diverse values of gap for MEMS comdrive-gaps between fingers and from fingers to the ground plane, many laborious works of FEM compared with those of BEM are needed because BEM can provide a complete solution in terms of boundary values only, with substantial saving in modeling effort. Therefore, there is no doubt that BEM has been become a very appealing approach in numerical simulation of MEMS [11] even if many engineers still use commercial package and waste much time to set up diverse FEM models during the variable design stage nowadays.

Although some simplified numerical models for electrostatic combdrive can be found in Refs. [4,5], there are still three types of fringing fields not taken into account, which result from the ground plane, widths and heights of fixed and movable fingers. In order to obtain more reasonable computational results for the electric field, the DBEM is employed and developed to analyze electrostatic problems for MEMS combdrive levitation considering the fringing of field around the edges, since the DBEM is a very efficient new method for solving the electrostatic problems of EM and MEMS [12,13]. After using DBEM to accurately calculate the electrostatic response of the comb finger biased with a dc voltage, the induced vertical force per unit length of the movable comb finger at different levitation positions can be obtained. In order to check the validity of the numerical model presented in this article, an example of in-plane interdigitated combdrive designs from Ref. [3] is furnished, and the solutions of DBEM are compared with analytical solutions if available and with a commercial FEM package [14].

As we know, with rapid increase of device density and working frequency in VLSI circuits, the electrical characteristics of interconnects are becoming more important factors governing the circuit performances such as delay, power consumption, reliability, etc. This has increased the interests in efficient methods for calculating electrical parameters of interconnects. Therefore, many researchers have used diverse accelerated BEMs (e.g. FastCap, GIMEI, ODDM, QMM..., etc.) for efficiently solving the large-scale problem of VLSI and EM systems [15-19]. To the best knowledge of authors, 
the accelerated BEMs are very efficient for large-scale problem because they can reduce CPU time and memory requirement from exponential order to logarithmic order. For a problem with $N$ degree of freedom, or $N$ unknowns, the accelerated BEMs use $\mathrm{O}(N \log N)$ memory, and close to $\mathrm{O}(N \log N)$ time, as opposed to the traditional methods requiring $\mathrm{O}\left(N^{2}\right)$ memory and $\mathrm{O}\left(N^{2}\right)$ time [20]. As $N$ becomes very large, there will be a tremendous disparity in memory and time usage between the accelerated BEMs and conventional BEMs. Although many BEM researchers have used diverse accelerated BEMs for large-scale problem (e.g. complex VLSL modeling) because they need to deal with the huge dense matrix from BEM. But for the concerned levitation problem of MEMS combdrive, it is not necessary to set up a huge BEM model because only the surface charge distribution around the boundary of movable finger needed. In reality, the accelerated BEM may have advantage over DBEM for large-scale problem, but this predominance is not obvious for the non-large-scale problem like the cases presented in this article because the model of MEMS combdrive is not so large as VLSI system. As the accelerated BEM can not display its advantage (computational efficiency) over the DBEM for non-large-scale problem, the DBEM was used in this article because it is an alternate efficient method for MEMS combdrive.

The paper is organized as follow. In Section 2, we concisely introduce the procedure of DBEM for electrostatic problems. Numerical results are provided and compared in Section 3 to establish the validity and accuracy of the DBEM and to study the effect of gap size variation for the levitation of MEMS combdrive. Some remarks based on the reported results were discussed in Section 4. Finally, there is a concise conclusion in Section 5.

\section{Dual integration equation for electrostatic problems}

For a homogeneous medium, the governing equation of electrostatics can be written in the following form

$\nabla^{2} V=-\rho / \varepsilon$

where $\nabla^{2}$ is the Laplacian operator. Eq. (1) is known as Poisson's equation; it states that the divergence of the gradient of electric potential (V) equals $-\rho / \varepsilon$ for a simple medium, where $\varepsilon$ is the permittivity of the medium and $\rho$ is the volume density of free charges [7]. At points in a simple medium where there is no free charge, Eq. (1) is reduced to

$\nabla^{2} V=0$

which is known as Laplace's equation. Eq. (2) plays a very important role in MEMS and EM. It is the governing equation for electrostatic problems involving a set of conductors, such as capacitors, maintained at different potentials. Once $V$ is found from Eq. (2), $E$ (electric field intensity) can be determined from $-\nabla V$, and the charge distribution on the conductor surfaces can be determined from $\rho_{\mathrm{s}}=\varepsilon E_{n}$.

Generally the electrostatic problem consists of finding the unknown potential function $\Phi$ (or $V$ ) in the partial differential equation. In addition to the fact that $\Phi$ satisfies $\nabla^{2} \Phi=0$ within a prescribed solution region $\boldsymbol{\Omega}$, the potential function $\Phi$ must satisfy certain conditions on $\mathbf{B}$ which is the boundary of $\boldsymbol{\Omega}$. Usually these boundary conditions are the Dirichlet $(\Phi(x)=f(x))$ and Neumann $\left(\partial \Phi(x) / \partial n_{x}=g(x)\right)$ types. Therefore, the governing equation of electrostatic problems could be written in the following form.

$\nabla^{2} \Phi(x)=0, x$ in $\mathbf{\Omega}$

where $f(x)$ and $g(x)$ denote known boundary data, and $n_{\mathrm{x}}$ is the unit outer normal vector at the point $x$ on the boundary $\mathbf{B}$.

Based on the dual boundary integral equation formulation for electrostatic problem [13], we have

$$
\begin{gathered}
\alpha \Phi(x)=\mathrm{CPV} \int_{B} T(\mathbf{s}, \mathbf{x}) \Phi(s) \mathrm{d} B(s) \\
-\mathrm{RPV} \int_{B} U(\mathbf{s}, \mathbf{x})\left[\partial \Phi(s) / \partial \mathbf{n}_{s}\right] \mathrm{d} B(s) \\
\alpha\left[\partial \Phi(x) / \partial n_{x}\right]=\operatorname{HPV} \int_{B} M(\mathbf{s}, \mathbf{x}) \Phi(s) \mathrm{d} B(s) \\
-\operatorname{CPV} \int_{B} L(\mathbf{s}, \mathbf{x})\left[\partial \Phi(s) / \partial \mathbf{n}_{s}\right] \mathrm{d} B(s)
\end{gathered}
$$

where the kernel functions, $U(\mathbf{s}, \mathbf{x})=\ln (r), T(s, x)=$ $\partial U(\mathbf{s}, \mathbf{x}) / \partial n_{s}, \quad L(\mathbf{s}, \mathbf{x})=\partial U(\mathbf{s}, \mathbf{x}) / \partial n_{x}, \quad M(\mathbf{s}, \mathbf{x})=\partial^{2} U(\mathbf{s}, \mathbf{x}) /$ $\partial n_{x} \partial n_{s}, r=|\mathbf{s}-\mathbf{x}|, \mathbf{s}$ and $\mathbf{x}$ being position vectors of the points $s$ and $x$, respectively, and $\mathbf{n}_{s}$ is the unit outer normal vector at point $s$ on the boundary (see Fig. 2). Table 1 shows the explicit forms of the four kernel functions $U(\mathbf{s}, \mathbf{x}), T(\mathbf{s}, \mathbf{x}), L(\mathbf{s}, \mathbf{x})$ and $M(\mathbf{s}, \mathbf{x})$. In addition, RPV is the Riemann Principal Value, CPV is the Cauchy Principal Value, HPV is the Hadamard Principal

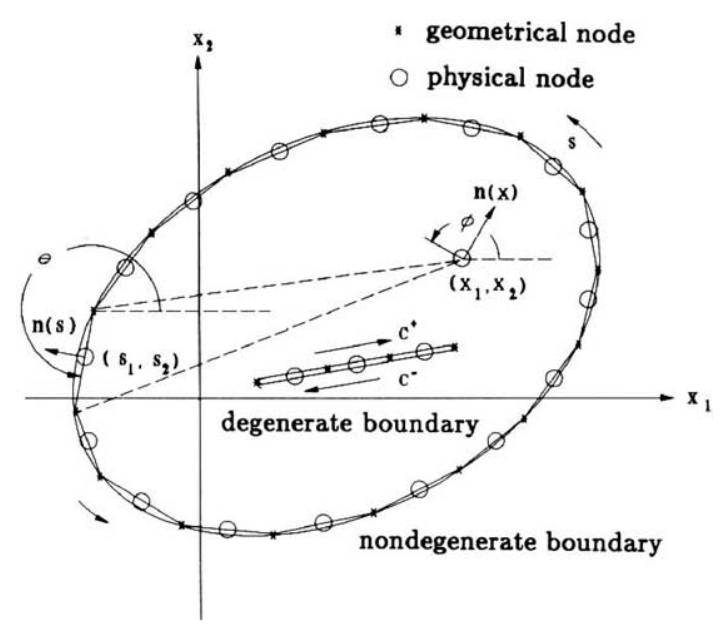

Fig. 2. Boundary element discretization for degenerate boundary and nondegenerate boundary. 
Table 1

The explicit forms of four kernel functions in dual integral equations

\begin{tabular}{lllll}
\hline Kernel function & $U(\mathbf{s}, \mathbf{x})$ & $T(\mathbf{s}, \mathbf{x})$ & $L(\mathbf{s}, \mathbf{x})$ & $M(\mathbf{s}, \mathbf{x})$ \\
\hline $\begin{array}{l}\text { Order of } \\
\text { singularity }\end{array}$ & Weak & Strong & Strong & Hypersingular \\
$\begin{array}{l}\text { Two-dimensional } \\
\text { case }\end{array}$ & $\ln (r)$ & $-y_{i} n_{i} / r^{2}$ & $y_{i} \bar{n}_{i} / r^{2}$ & $\begin{array}{l}2 y_{i} y_{j} n_{i} \bar{n}_{j} / r^{4} \\
-n_{i} \bar{n}_{i} / r^{2}\end{array}$ \\
$\begin{array}{l}\text { Three-dimensional } \\
\text { case }\end{array}$ & $-1 / r$ & $-y_{i} n_{i} / r^{3}$ & $y_{i} \bar{n}_{i} / r^{3}$ & $\begin{array}{l}3 y_{i} y_{j} n_{i} \bar{n}_{j} / r^{5} \\
\text { Remark }\end{array}$ \\
\hline
\end{tabular}

Value, and $\alpha$ depends on the collocation point $(\alpha=2 \pi$ for an interior point, $\alpha=\pi$ for a smooth boundary, $\alpha=0$ for an exterior point). The commutativity property of the trace operator and the normal derivative operator provides us with alternative ways to calculate the Hadamard principal value analytically. Generally, Eq. (4) is called singular boundary integral equation, and Eq. (5) is called hypersingular boundary integral equation. Since the hypersingular boundary integral equation plays an important role in the degenerate problems, many researchers have paid much attention to
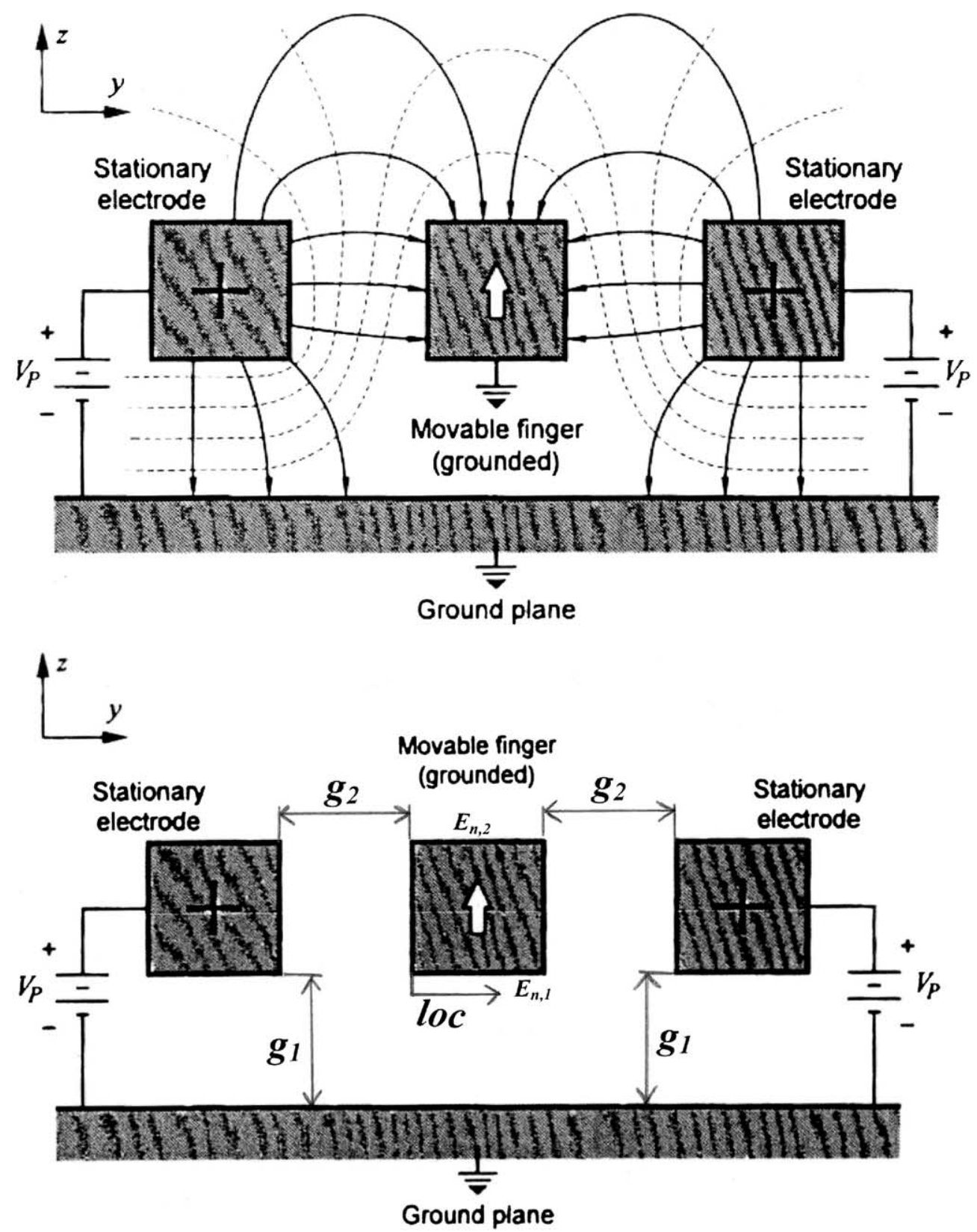

Fig. 3. Cross-section of the potential contours (dashed lines) and the electric fields (solid lines) of a comb finger under levitation force induced by two adjacent electrodes biased at a positive potential. 
this. After discretizing the boundary into $2 \mathrm{~N}$ boundary elements, Eqs. (4) and (5) reduce to

$[U]_{2 N \times 2 N}\{t\}_{2 N \times 1}=[T]_{2 N \times 2 N}\{u\}_{2 N \times 1}$

$[L]_{2 N \times 2 N}\{t\}_{2 N \times 1}=[M]_{2 N \times 2 N}\{u\}_{2 N \times 1}$

where $[U],[T],[L]$ and $[M]$ are the four influence matrices, $\{u\}$ and $\{t\}$ are the boundary data for the primary and the secondary boundary variables, respectively. Generally for electrostatic problems without singularities arising from degenerate scale, the aforementioned influence matrix $[U]$ is nonsingular, either Eqs. (6) or Eq. (7) can be solved by Gaussian elimination and LU decomposition very well. But for degenerate scale problem, $[\mathrm{U}]$ matrix is singular and the rank is deficient, then some regularization BEM in conjunction with SVD (singular value decomposition) technique could be used [21].

\section{DBEM simulation for the electrostatic field of MEMS combdrive levitation}

The successful electrostatic actuation of micromechanical structures requires a ground plane under the structure in order to shield it from relatively large vertical fields [3]. In order to demonstrate the suitability of DBEM presented in this article, an electrostatic combdrive problem proposed by Tang et al. [3] was used (see Case 1). In this case, a $4 \mu \mathrm{m}$-wide $\times 2 \mu \mathrm{m}$-thick comb finger excited by two identically sized electrodes situated $2 \mu \mathrm{m}$ away from both sides of the finger, and $2 \mu \mathrm{m}$ above a grounded substrate was used (see Fig. 3). The following Case 2 was used to study the effect of the gap size variation (from 2.0 to $0.4 \mu \mathrm{m})$ between combdrive fingers and ground plane $\left(g_{1}\right)$ for levitation of MEMS comdrive, but the values of gap between movable finger and fixed finger $\left(g_{2}\right)$ are still confined to $2.0 \mu \mathrm{m}$. In Case 3, we intend to investigate the effect of the size variation of $g_{2}$ (from 5.0 to $0.5 \mu \mathrm{m}$ ), but the values of $g_{1}$ are still confined to $2.0 \mu \mathrm{m}$.

Case 1: A comb finger under levitation force induced by two adjacent electrodes biased at a positive potential $V_{p}$ shown in Fig. 3. In order to check the accuracy if using DBEM, we will determine the electric potential distribution.

From Fig. 3, one can see that there is an obvious fringing of field around the edges of fixed finger and movable finger, and the physical behavior (e.g. electric potential and electric field intensity) of this area is very complicated. Since it is not easy to obtain the analytical solutions, and some simplified numerical models for electrostatic combdrives from Refs. [4,5] cannot accurately simulate the fringing field, the FEM simulation [14] was used to compare with the following DBEM data. Because of the fringing of field around the edges, a large finite element model was set up in order to obtain a reasonable result. In addition, the symmetric boundary between two adjacent fingers using

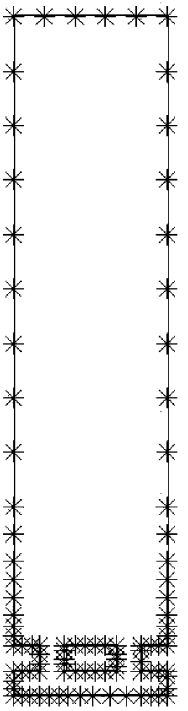

Fig. 4. The related DBEM coarse mesh discretization.

proper Neumann boundary condition was used to simplify the dimension of FEM and DBEM models.

Over three thousand points will be analyzed using coarse mesh discretization (95 elements and 95 nodes; see Fig. 4) of DBEM, and compared with reference data computed from a large refined mesh FEM model (3608 elements and 3790 nodes; see Fig. 5a) because the results from the coarser mesh FEM model (1490 elements and 1607 nodes; see Fig. 5b) are not adequately accurate. The results of electric potential under refined mesh FEM and coarse mesh DBEM

(a)
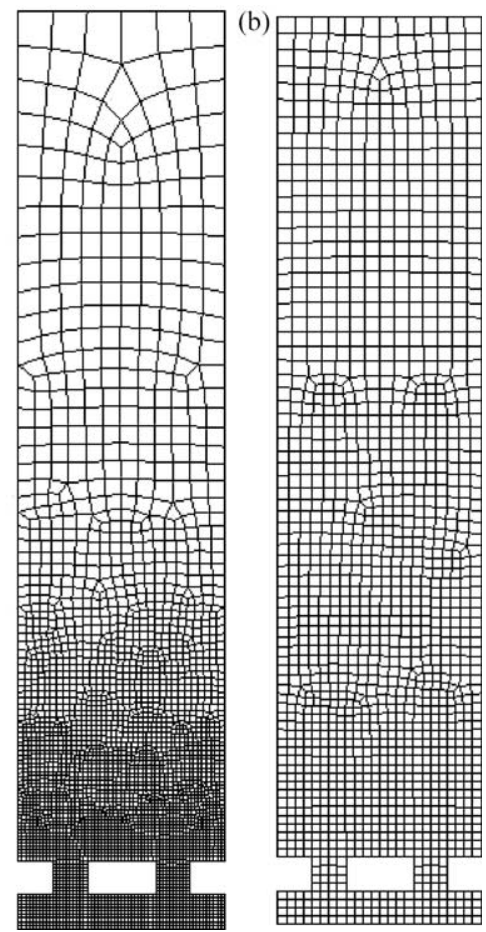

Fig. 5. The related FEM mesh discretization (Left part: refined mesh model; Right part: coarser mesh model). 

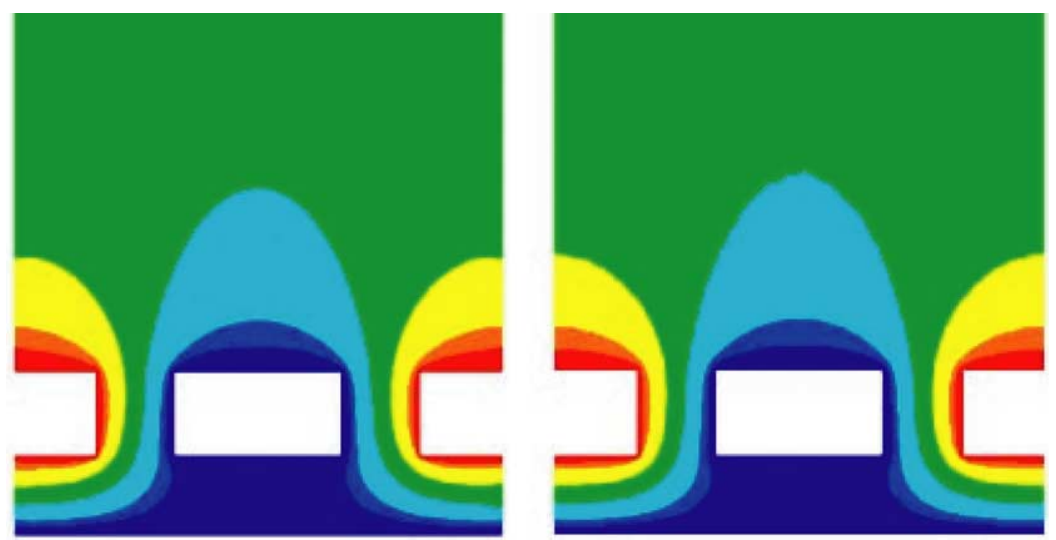

Fig. 6. Results of electric potential field (equipotential lines-Red color: $+V_{p}$; Blue color: 0 ) of combdrive using coarse mesh DBEM (Left part) and refined mesh FEM (Right part) (For interpretation of the references to colour in this figure legend, the reader is referred to the web version of this article).

were shown in Fig. 6. Comparing the results of electric potential field using coarse mesh DBEM and refined mesh FEM, one can see that the difference of electric potential distribution listed in Table 2 is very little $(<1.3 \%)$, hence the DBEM used in this article is an efficient method for solving electrostatic MEM combdrive problems. From Fig. 6, the ground plane contributes to an obvious unbalanced electrostatic field distribution if a heavily doped polysilicon film underlies the resonator and the comb structure like the rough sketch map shown in Fig. 3.

Case 2: Because $g_{1}$ can play a very important role for the levitation of MEMS combdrive, the effect of those will be investigated in the following case. Similar to the aforementioned case, the values of $g_{2}$ are still confined to $2.0 \mu \mathrm{m}$, but $g_{1}$ are variable from 2.0 to $0.4 \mu \mathrm{m}$. If a movable comb finger when differential dc bias $V_{p}$ is applied to the two adjacent electrodes shown in Fig. 3, determine the distribution of normal electric field intensity $\left(E_{n}\right)$ on the bottom and upper side of movable finger under diverse values of $g_{1}$. Besides $E_{n}$, also calculate the levitating force density $F$ acting on the movable finger.

Because many FEM models need to be established for diverse gap variation, FEM is not a good choice for Case 2, so we use DBEM to perform the following tasks since the discretizations of DBEM are restricted only to the boundaries, making data generation much easier than FEM. By way of the DBEM, the distribution of normal electric field intensity $\left(E_{n}\right)$ on the bottom and upper side of movable finger under diverse values of $g_{1}$ were shown in Figs. 7 and 8, respectively. From Fig. 7, one can see that the values of normal electric field intensity $\left(E_{n, 1}\right)$ on the bottom of movable finger are obviously dependent on the values of $g_{1}$ and the location to the left side of movable finger (loc). Unlike $E_{n, 1}$, the values of normal electric field intensity $\left(E_{n, 2}\right)$ on the upper side of movable finger are only apparently counting on the value of loc, and the effect of the values of $g_{1}$ can be ignored (see Fig. 8). Because the charge distribution on the conductor surfaces can be determined from $\rho_{s}=\varepsilon E_{n}$ (The normal component of the electric field $E_{n}$ at a conductor boundary is equal to the surface charge density $\rho_{s}$ on the conductor divided by the permittivity $\varepsilon$ [7]) if $\varepsilon$ is a constant, the relationship between the normal force density $f_{n}$ acting on the surface of a conductor and the charge density $\rho_{s}$ of that conductor is

$f_{n}=-0.5 \rho s^{2} / \varepsilon$

Thus, the electrostatic force density $F_{n}$ acting on the movable finger along the boundary

$F_{n}=\int_{B} f \mathrm{~d} B$

can be calculated if $\rho_{s}$ (or $E_{n}$ ) is known. Therefore, the levitating force density $F$ (normal to the substrate) acting on each movable finger is equal to the difference of electrostatic force density $F_{n}$ between upper side and bottom of concerned movable finger, and that is obviously dependent on the difference of $E_{n}$ between upper side and bottom of movable finger. Because the difference of $F_{n}$ and $E_{n}$ between upper side and bottom of concerned movable finger is obvious under diverse values of $g_{1}$, the levitating force density $F$ acting on the movable finger shown in Fig. 9 does apparently rely on the variation of $g_{1}$ if the values of $g_{2}$ are still confined to a constant value. Results also show that the less the values of $g_{1}$ are, the larger the levitating force density $F$ is.

Case 3: Besides the effect of $g_{1}$, the values of $g_{2}$ also master greatly on the levitating force. Not alike Case 2,

Table 2

The results of electric potential under DBEM and FEM

\begin{tabular}{llll} 
Locations $(y, z)$ & $V(y, z)$ from DBEM & $V(y, z)$ from FEM & Difference (\%) \\
\hline$(5.9867,5.0151)$ & $0.1580444 V_{p}$ & $0.15975 V_{p}$ & +1.08 \\
$(6.1889,4.8152)$ & $0.1301514 V_{p}$ & $0.13162 V_{p}$ & +1.13 \\
$(5.7961,4.4058)$ & $0.06673584 V_{p}$ & $0.067443 V_{p}$ & +1.06 \\
$(1.6000,1.6000)$ & $0.0748376 V_{p}$ & $0.073904 V_{p}$ & -1.25 \\
\hline
\end{tabular}

The origin $(x, 0,0)$ of the coordinates is located at the left bottom corner of DBEM model shown in Fig. 4. 


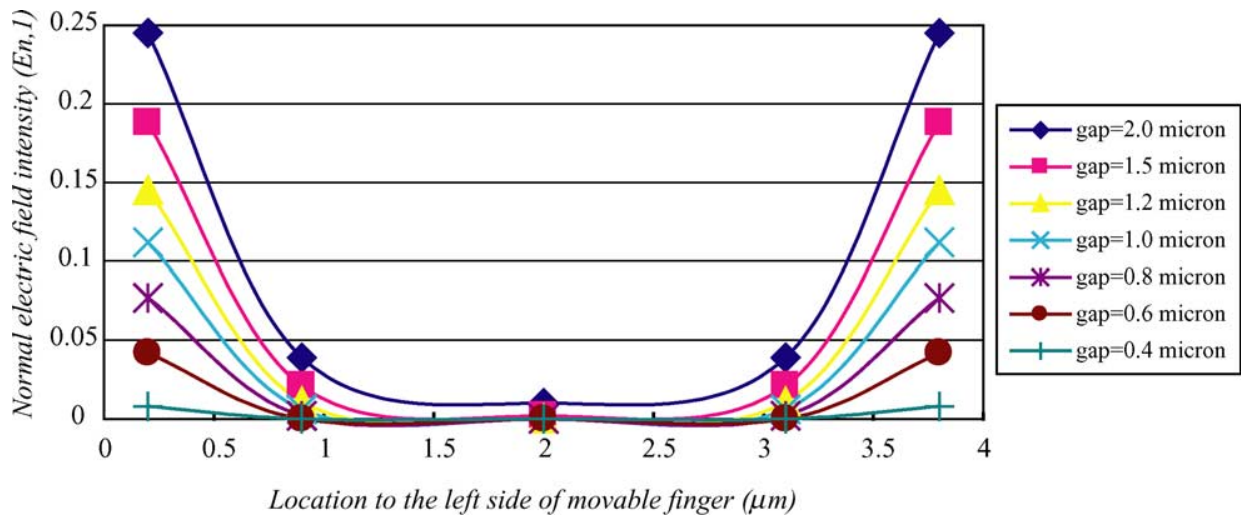

Fig. 7. The distribution of normal electric field intensity $\left(E_{n, 1}\right)$ on the bottom of movable finger under diverse gaps between combdrive fingers and ground plane $\left(g_{1}\right)$. (unit: $V_{p} / \mu \mathrm{m}$ ).

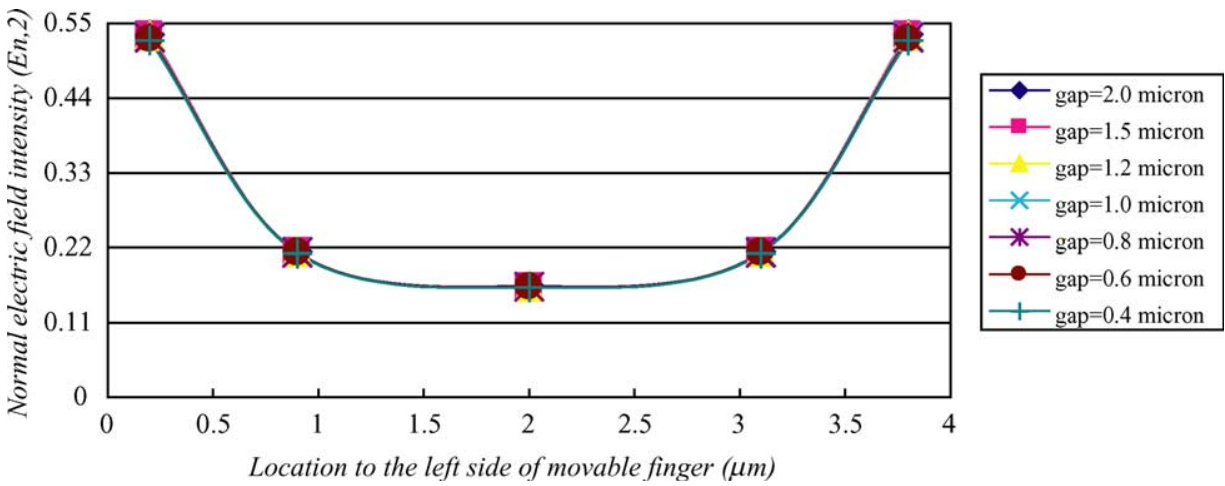

Fig. 8. The distribution of normal electric field intensity $\left(E_{n, 2}\right)$ on the upper side of movable finger under diverse values of $g_{1}$. (unit: $\left.V_{p} / \mu \mathrm{m}\right)$.

the values of $g_{2}$ are variable from 5.0 to $0.5 \mu \mathrm{m}$, but the values of $g_{1}$ are still confined to $2.0 \mu \mathrm{m}$. If a movable comb finger when differential dc bias $V_{p}$ is also applied to the two adjacent electrodes shown in Fig. 3, determine the distribution of normal electric field intensity $\left(E_{n}\right)$ on the bottom and upper side of movable finger under diverse values of $g_{2}$, and also calculate the levitating force density $\mathrm{F}$ acting on the movable finger.

Similar to Case 2, the DBEM was used since their discretizations are only confined to the boundaries. By way of the DBEM, the distribution of $E_{n}$ on the bottom and upper side of movable finger under diverse values of $g_{2}$ were shown

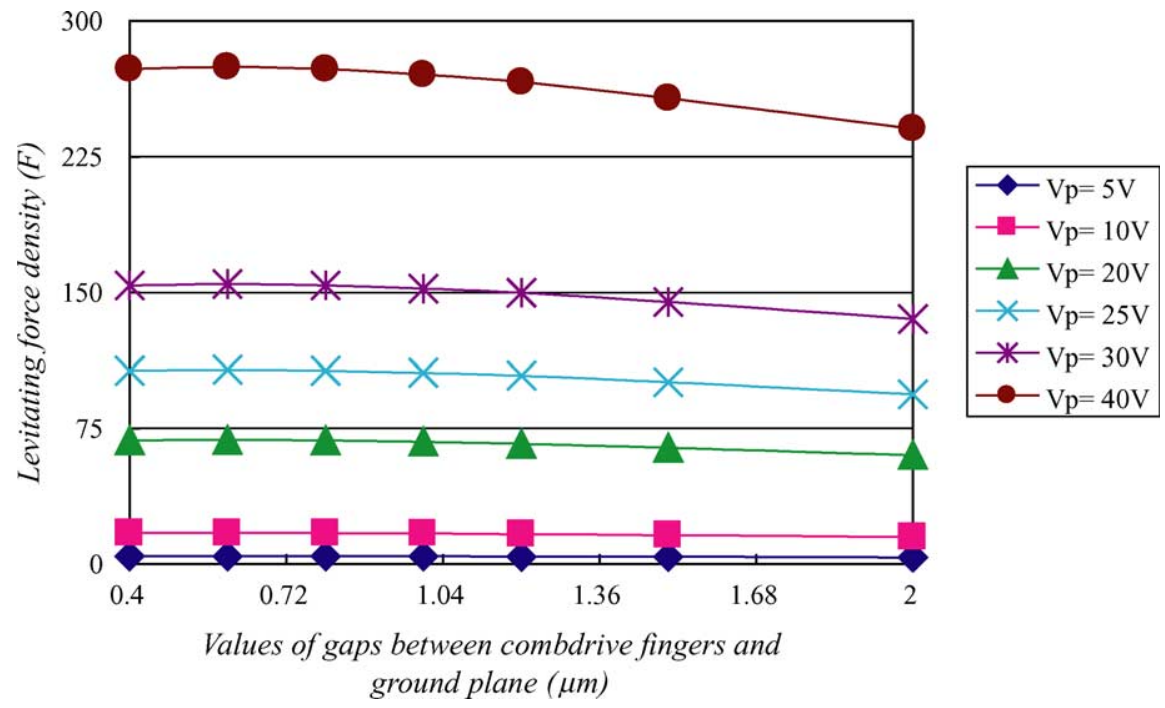

Fig. 9. The levitating force density $(F)$ acting on the movable finger under diverse values of $g_{1}$ and $V_{p}$. (unit: $\varepsilon V^{2} / \mu m$ ). 

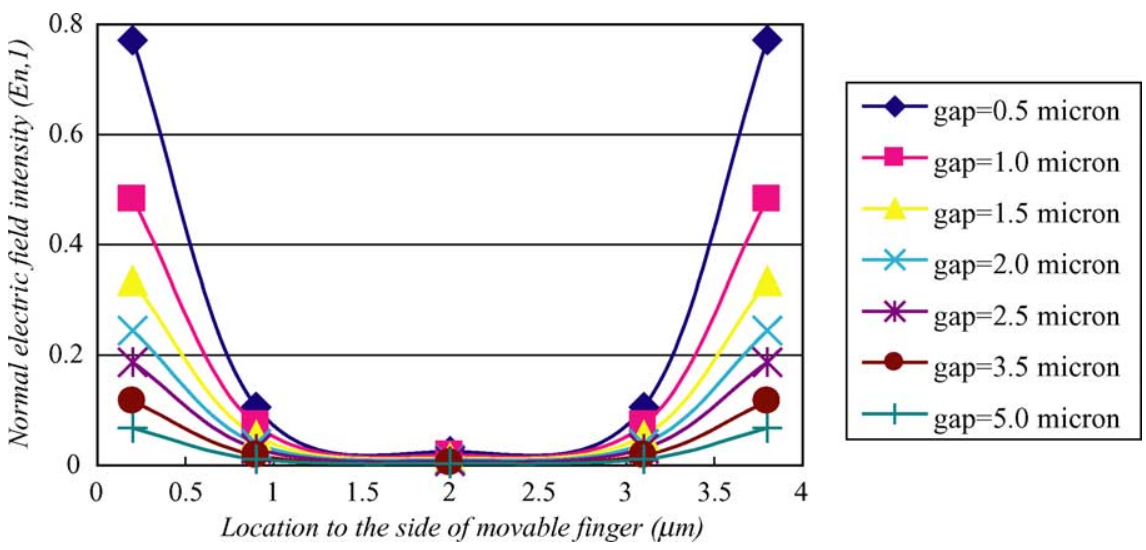

Fig. 10. The distribution of $E_{n, 1}$ on the bottom of movable finger under diverse gaps between movable finger and fixed finger $\left(g_{2}\right)$. (unit: $\left.V_{p} / \mu \mathrm{m}\right)$.

in Figs. 10 and 11, respectively. From Figs. 10 and 11, one can see that the values of $E_{n, 1}$ on the bottom and $E_{n, 2}$ on the upper side of movable finger are both obviously dependent on the values of $g_{2}$ and the value of loc. Unlike the results of Case 2 shown in Fig. 8, the values of $E_{\mathrm{n}, 2}$ are not only apparently counting on the value of loc, but also the values of $g_{2}$ (see Fig. 11). Because the difference of $F_{n}$ and $E_{n}$ between upper side and bottom of concerned movable finger is also notable under diverse values of $g_{2}$, the levitating force density $F$ acting on the movable finger shown in Fig. 12 does

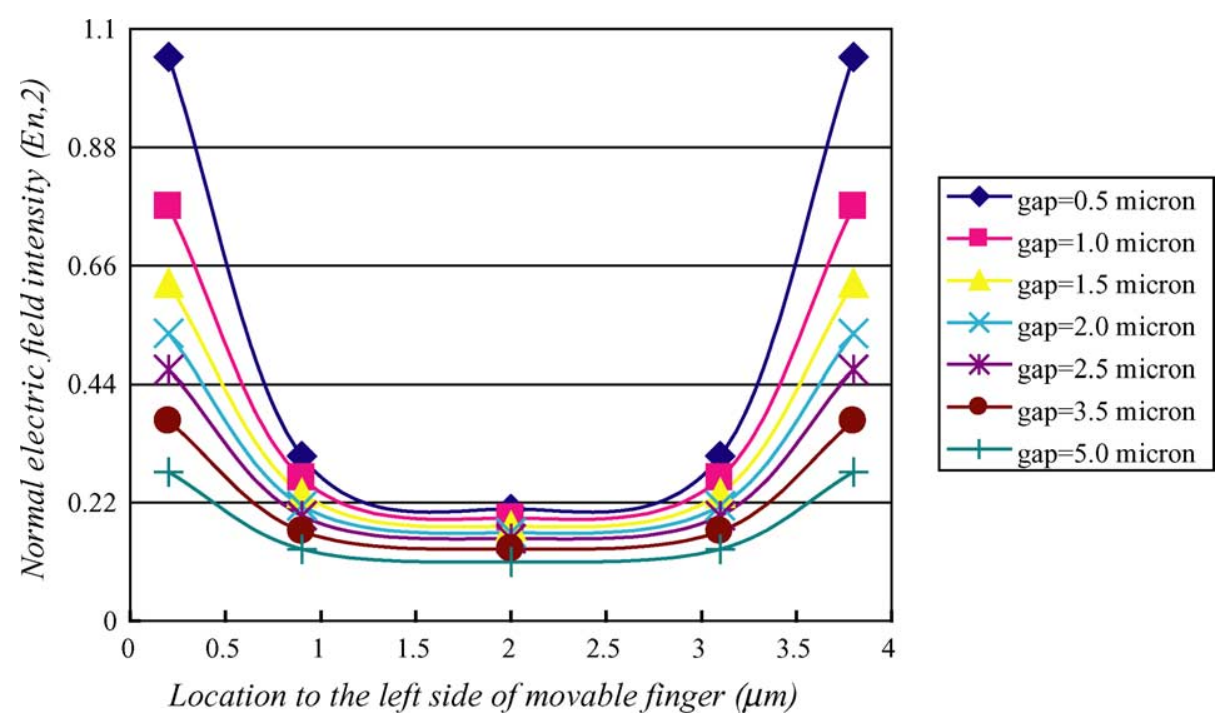

Fig. 11. The distribution of $E_{n, 2}$ on the upper side of movable finger under diverse values of $g_{2}$. (unit: $V_{p} / \mu \mathrm{m}$ ).

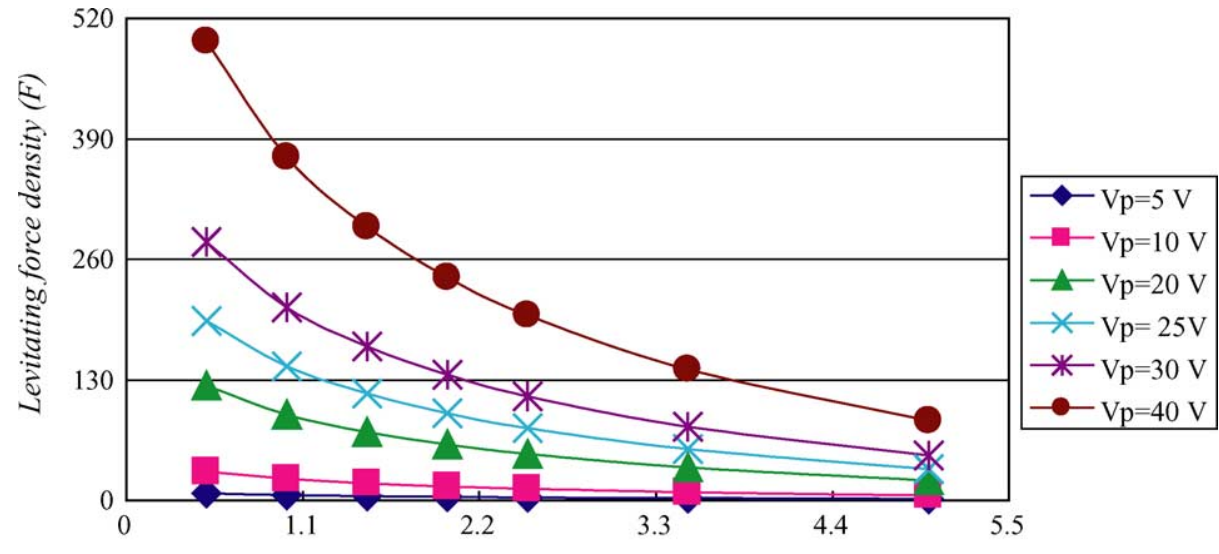

Values of gap between movable finger and fixed finger $(\mu \mathrm{m})$

Fig. 12. The levitating force density $(F)$ acting on the movable finger under diverse values of $g_{2}$ and $V_{p}$. (unit: $\varepsilon V^{2} / \mu \mathrm{m}$ ). 
apparently rely on the variation of $g_{2}$ if the values of $g_{1}$ are still confined to a constant value. Results also show that the less the values of $g_{2}$ are, the larger the levitating force density $F$ is.

\section{Discussions}

1. Because the control of levitating force can get higher quality factor of combdrive, how to get the accurate electrostatic field is very important and indispensable for the design of MEMS devices. After using coarse mesh DBEM presented in this article to accurately calculate the electrostatic response of the comb finger biased with a dc voltage, the induced vertical force per unit length of the movable comb finger at different levitation positions can be obtained. Results show that the less the gaps between combdrive fingers and ground plane $\left(g_{1}\right)$ are, the larger the levitating force density $(F)$ acting on the movable finger is. In addition, the levitating force becomes more predominant as the gaps between movable finger and fixed finger $\left(g_{2}\right)$ decrease.

2. If the values of $g_{2}$ are constant and the values of $g_{1}$ are variable like Case 2, the values of $E_{n, 1}$ on the bottom of movable finger are obviously dependent on the values of $g_{1}$ and the location to the left side of movable finger (loc), but the values of $E_{n, 2}$ on the upper side of movable finger are only apparently counting on the value of $l o c$, and the effect of the values of $g_{1}$ can be ignored. If the values of $g_{2}$ are variable but the values of $g_{1}$ are still confined like Case 3 , the values of $E_{n, 1}$ on the bottom and $E_{n, 2}$ on the upper side of movable finger are both obviously dependent on the values of $g_{2}$ and the value of $l o c$.

3. By comparing the element mesh of refined mesh FEM and coarse mesh DBEM of electrostatic combdrive considering the fringing of field around the edges, one can see that numbers of elements and nodes for refined mesh FEM are much higher than those of coarse mesh DBEM to get a reasonable result. Though using FEM was widespread for MEMS device nowadays, it is still very difficult to establish the boundary conditions and generate the all proper FEM because the values of gap for MEMS comdrive-gaps between fingers $\left(g_{2}\right)$ and from fingers to the ground plane $\left(g_{1}\right)$ always change many times before final layout in the variable design stage. Therefore, we strongly recommend the DBEM for studying the effect of gap size variation for the levitation of MEMS combdrive because the DBEM's discretizations are restricted only to the boundaries, and it's making data generation much easier than FEM.

\section{Conclusions}

The dual integral formulation of electrostatic combdrive problems considering the fringing of field around the edges has been presented in this article. Comparisons of the results between FEM and DBEM analyses were discussed with respect to diverse gap size for electrostatic MEMS combdrive in order to demonstrate the efficiency of DBEM. It has been ensured that the capabilities of coarse mesh DBEM simulation are acceptable after comparison with the refined mesh FEM data. For electrical engineering practices, since the numbers of elements and nodes for refined mesh FEM are much higher than those of coarse mesh DBEM to get a reasonable result, and it wastes much time for diverse values of gap design if using the domaintype FEM, so the present boundary-type DBEM has great potential for industrial applications, especially in the initial variable design stage.

\section{References}

[1] W.C. Tang, T.C.H. Nguyen, M.W. Judy, R.T. Howe, Lateral driven polysilicon resonant microstructures, Sensors Actuators 20 (1989) 25-32.

[2] W.C. Tang, T.C.H. Nguyen, M.W. Judy, R.T. Howe, Electrostaticcomb drive of lateral polysilicon resonators, Sensors Actuators A2123 (1990) 328-331.

[3] W.C. Tang, M.G. Lim, R.T. Howe, Electrostatic comb drive levitation and control method, IEEE J. Microelectromech. Syst. 1 (4) (1992) $170-178$.

[4] J.L.A. Yeh, C.Y. Hui, N.C. Tien, Electrostatic model for an asymmetric combdrive, IEEE J. Microelectromech. Syst. 9 (1) (2000) 126-135.

[5] W.A. Johnson, L.K. Warne, Electrophysics of micromechanical comb actuators, IEEE J. Microelectromech. Syst. 4 (1) (1995) 49-59.

[6] W. Ye, S. Mukherjee, N.C. MacDonald, Optimal shape design of an electrostatic comb drive in microelectromechanical systems, IEEE J. Microelectromech. Syst. 7 (1) (1998) 16-26.

[7] D.K. Cheng, Field and Wave Elctromagnetics, Addison-Wesley, Reading, MA, 1989.

[8] U. Beerschwinger, N.G. Miline, S.J. Yang, R.L. Reuben, A.J. Sangster, H. Ziad, Coupled electrostatic and mechanical FEA of a micromotor, IEEE J. Microelectromech. Syst. 3 (4) (1994) $162-171$.

[9] P.P. Silvester, F.L. Ferrari, Finite Elements for Electrical Engineers, Cambridge University Press, Cambridge, 1983.

[10] J. Wiersig, Boundary element method for resonances in dielectric microcavities,, J. Opt. A: Pure Appl. Opt. 5 (2003) 53-60.

[11] S.D. Senturia, N. Aluru, J. White, Simulating the behavior of MEMS devices: computational methods and needs, IEEE Comput. Sci. Engng Mag. January-March (1997) 30-43.

[12] S.W. Chyuan, Y.S. Liao, J.T. Chen, An efficient method for solving electrostatic problems, IEEE Comput. Sci. Engng Mag. May-June (2003) 52-58.

[13] Y.S. Liao, S.W. Chyuan, J.T. Chen, An alternatively efficient method for simulating the electrostatic field and levitating force of MEMS combdrive, J. Micromech. Microengng 14 (2004) 1258-1269.

[14] I-DEAS User's Guide, Finite Element Modeling, SDRC, 1990.

[15] K. Nabors, J.K. White, Multipole-accelerated capacitance extraction algorithrms for 3-D structures with multiple dielectrics, IEEE Trans. Circuits Syst. 39 (1992) 946-954. 
[16] W. Yu, Z. Wang, J. Gu, Fast capacitance extraction of actual 3-D VLSI interconnects using quasi-multiple medium accelerated BEM, IEEE Trans. Microwave Theory Tech. 51 (2003) 109-119.

[17] J.R. Phillips, J.K. White, A precorrected-FFT method for electrostatic analysis of complicated 3-D structures, IEEE Trans. Computer-Aid. Des. 16 (1997) 1059-1072.

[18] C.Y. Leung, S.P. Walker, Iterative solution of large three-dimensional BEM elastostatic analysis using the GMRES technique, Int. J. Numer. Meth. Engng 40 (1997) 2227-2236.

[19] J.T. Chen, K.H. Chen, Applications of the dual integral formulation in conjunction with fast multipole method in large-scale problems for 2D exterior acoustics, Engng Anal. Bound. Elem. 28 (2004) 685-709.

[20] W.C. Chew, H.Y. Chao, T.J. Cui, C.C. Lu, S. Ohnuki, Y.C. Pan, J.M. Song, S. Velamparambil, J.S. Zhao, Fast integral equation solvers in computational electromagnetics of complex structures, Engng. Anal. Bound. Elem. 27 (2003) 803-823.

[21] J.T. Chen, S.R. Lin, On the rank-deficiency problems in boundary integral formulation using the Fredholm alternative theory and singular value decomposition technique, Proceedings of Fifth World Congress on Computational Mechanics, July 7-12, 2002, Vienna, Austria. 